\title{
Высоковольтные диффузионные диоды с резким восстановлением. І. Численное моделирование
}

\author{
(C) А.C. Кюрегян \\ ПАО «НПО „Электромодуль“», \\ 109052 Москва, Россия \\ E-mail: semlab@yandex.ru
}

Поступила в Редакцию 27 декабря 2018 г. В окончательной редакции 18 января 2019 г. Принята к публикации 29 января 2019 г.

\begin{abstract}
Методами имитационного компьютерного моделирования впервые детально исследована работа диффузионных диодов с резким восстановлением в качестве прерывателей тока в мощных генераторах наносекундных импульсов. Указано одно из необходимых условий, минимизирующих потери в диффузионных диодах с резким восстановлением. Получены зависимости напряжения предимпульса, длительности фронта, амплитуды, длительности импульса, формируемого на активной нагрузке, и энергии коммутационных потерь в диффузионных диодах с резким восстановлением от площади прибора и плотности обрываемого тока. Показано, что результаты моделирования описываются полученными во второй части работы простыми аналитическими формулами с точностью $10-20 \%$, если амплитуда импульса не превосходит напряжение лавинного пробоя диффузионных диодах с резким восстановлением. Предложен обобщенный показатель качества диффузионных диодов с резким восстановлением, который можно использовать для оптимизации его параметров, режима работы и сравнения эффективности прерывателей тока различных типов.
\end{abstract}

Ключевые слова: прерыватели тока, диффузионные диоды с резким восстановлением, компьютерное моделирование, показатель качества.

DOI: 10.21883/FTP.2019.07.47877.9055

\section{1. Введение}

Высоковольтные диоды с резким восстановлением блокирующей способности (ДРВ) давно используются в качестве прерывателей тока в мощных генераторах наносекундных импульсов с промежуточным индуктивным накопителем энергии [1-3]. В общем случае ДРВ представляют собой полупроводниковые $p^{+}-p-n-n^{+}$структуры, которые, начиная с пионерской работы [4], изготавливались по обычной диффузионной технологии мощных кремниевых выпрямительных диодов [4-11]. В статье [8] было показано, что наличие $p$-слоя, сформированного путем диффузии $\mathrm{Al}$ на большую ( 100 мкм) глубину сушественно уменьшает время обрыва тока, а авторы недавней статьи [12] даже назвали такой $p$-слой „принципиальной особенностью конструкции этих диодов“. Однако в большинстве опубликованных статей, посвященных теоретическому анализу работы ДРВ, использовалась модель приборов с однородно легированными высокоомными $p$ - и(или) n-слоями [2-4,13-19], которые можно изготовить с помощью эпитаксиальной технологии [20] (далее для таких приборов мы используем аббревиатуру эДРВ). Лишь в трех работах [21-23] приведены некоторые результаты численного моделирования процессов в диффузионных ДРВ (далее - дДРВ ${ }^{1}$ )

\footnotetext{
${ }^{1}$ Получившее широкое распространение название ,дрейфовый диод с резким восстановлением (ДДРВ)“, впервые использованное в работе [5], представляется очень неудачным по двум причинам. Во-первых, оно не отражает технологию изготовления приборов. Во-вторых, приближение обратимого во времени амбиполярного дрейфа, использованное в работах $[2,5]$ и отраженное в этом названии, на самом деле
}

с толстым неоднородно легированным p-слоем, но в них не проводился детальный теоретический анализ процессов восстановлением блокирующей способности и обрыва тока, необходимый для проектирования приборов и расчета их предельно достижимых параметров.

Решению этой проблемы посвящена настоящая работа, которая состоит из двух частей. В первой части описаны эквивалентная схема генератора импульсов на основе ДРВ, использованная при численном моделировании и конструкция исследованных $p^{+}-p-n-n^{+}$-структур (разд. 2), изложены результаты численного моделирования работы дДРВ, которое выполнялось с помощью программы „Исследование“ [24] (разд. 3). Для анализа этих результатов использована простейшая аналитическая теория дДРВ, изложенная во второй части работы [25]. Ссылки на формулы с номерами $\mathrm{N}$ из работы [25] даются в формате (II.N). В заключение предложен обобщенный показатель качества ДРВ и приведены его зависимости от плотности обрываемого тока для исследованных дДРВ.

\section{2. Объекты исследования}

Каждый цикл коммутации ДРВ состоит из трех стадий [1-3]:

- стадия прямой накачки (СПН) длительностью $T_{f}$, во время которой слабо легированные $p$ - и $n$-слои заполняются неравновесной квазинейтральной электроннодырочной плазмой;

неприменимо для описания процесса восстановления блокирующей способности ДРВ. 
- стадия высокой обратной проводимости (СВОП) длительностью $t_{r}=T_{b}-T_{f} \leq T_{f} / 2$, во время которой неравновесная плазма вытягивается из $p$ - и $n$-слоев нарастающим обратным током $I$ индуктивного накопителя и ДРВ восстанавливает свою блокирующую способность в момент времени $t=T_{b}$;

- стадия обрыва тока (СОТ), во время которой практически весь ток перебрасывается из ДРВ в параллельную ему нагрузку $R$.

Для реализации такого цикла работы ДРВ было предложено много схем генератора, наиболее универсальная из которых изображена на рис. 1. Контуры $\left(C_{f}-L_{f}-S R D-S W_{1}\right)$ и $\left(C_{r}-L_{r}-S R D-S W_{2}\right)$ прямой и обратной накачки могут работать совместно (в этом случае ключи $S W_{1}$ и $S W_{2}$ замыкаются последовательно при $t=0$ и $t=T_{f} \approx \pi \sqrt{L_{f} C_{f}}$, когда токи через них равны нулю) или независимо (в этом случае ключ $S W_{1}$ замыкается при $t=0$ и размыкается при $t=T_{f}$, а ключ $S W_{2}$ замыкается при $t=T_{f}$ ), обеспечивая разнообразные режимы накачки. Именно этот последний вариант был использован в настоящей работе. Кроме того, для простоты мы считали, что прерыватель тока состоит из одного кристалла, хотя на практике обычно используют сборку из $m \gg 1$ последовательно соединенных кристаллов [1-3]. Для этого случая результаты настоящей работы следует пересчитать, увеличив все напряжения, сопротивления и индуктивности в $m$ раз, а емкости уменьшив в $m$ раз.

Исследовались дДРВ, изготовленные путем двухступенчатой диффузии акцепторов и доноров в пластины нелегированного кремния толщиной $d$. Сначала формируются толстые относительно слабо легированные $p$ - и $n$-слои с гауссовыми распределениями примесей, т. е.

$$
\begin{aligned}
& N_{a}(x)=N_{0} \exp \left(\frac{-2 l_{p} x-x^{2}}{\lambda_{p}^{2}}\right), \\
& N_{d}(x)=N_{0} \exp \left(\frac{-2 l_{n} x-x^{2}}{\lambda_{n}^{2}}\right),
\end{aligned}
$$

$\lambda_{n, p}=l_{n, p}\left(\ln N_{s n, s p} / N_{0}\right)^{-1 / 2}, \quad N_{s n, s p}-$ поверхностные концентрации доноров и акцепторов, $l_{p}$ и $l_{n}=\left(d-l_{0}\right)-$ расстояния от поверхностей кристалла до плоскости $p$-n-перехода $x=0$, где $N_{a}=N_{d}=N_{0}$. После этого

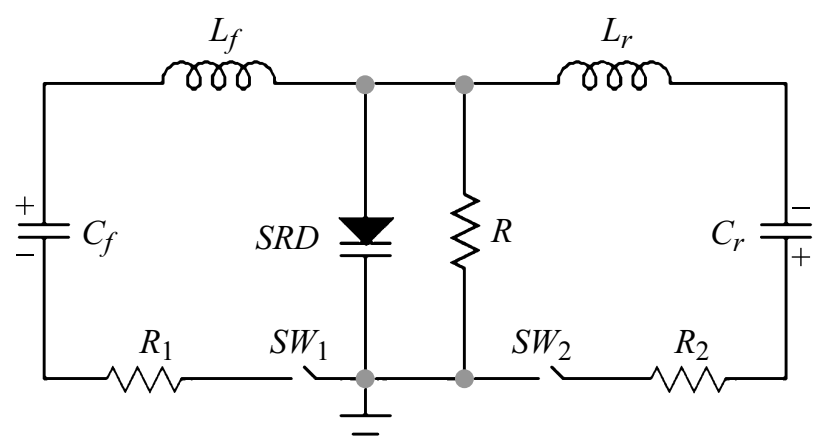

Рис. 1. Двухконтурная схема генератора импульсов на основе ДРВ (SRD) [2]. $R-$ сопротивление нагрузки, $R_{1,2}-$ сопротивления ключей $S W_{1,2}$. формируются тонкие $p^{+}$- и $n^{+}$-слои, достаточно сильно легированные для обеспечения эффективной инжекции во время СПН.

Как видно, количество свободных параметров, определяющих работу генераторов на основе дДРВ, столь велико (более десятка), что ограниченный объем статьи не позволяет изложить результаты полного анализа взаимосвязей параметров контура, дДРВ и импульса напряжения, формируемого на нагрузке. Поэтому мы вынуждены изложить результаты лишь для одного типичного варианта конструкции, которые тем не менее дают возможность прояснить всю необходимую физику, определяющую основные характеристики дДРВ. А именно, если это специально не оговорено, предполагаются следующие значения параметров дДРВ и контура: $d=200 \mathrm{м \kappa м}, N_{s n}=N_{s p}=5 \cdot 10^{16} \mathrm{~cm}^{-3}, N_{0}=6 \cdot 10^{13} \mathrm{~cm}^{-3}$, $l_{p}=120-150$ мкм, время жизни электронов и дырок $\tau_{n o}=\tau_{p o}=10$ мкс и $R=1$ Ом, $C_{r}=2 C_{f}=2$ мкФ, $L_{r}=L_{f} / 2=4 \mathrm{н} \Gamma_{\mathrm{H}}, R_{1}=R_{2}=1$ мОм. Напряжение пробоя $U_{B}$ таких дДРВ изменялось в пределах $850-830 \mathrm{~B}$, а длительности СПН и СВОП без учета потерь в контуре и ДРВ $T_{f}=2 t_{r}=\pi \sqrt{L_{r} C_{r}} \approx 281$ нс. Начальные напряжения $U_{C f}, U_{\mathrm{Cr}}$ на конденсаторах $C_{f}, C_{r}$ были равны по величине и противоположны по знаку.

\section{3. Численное моделирование дДРВ}

Процесс эволюции электронно-дырочной плазмы и напряженности электрического поля во время СВОП был подробно описан в работах $[26,27]$. Он начинается с восстановления $p^{+}-p$ - и $n-n^{+}$-переходов и последующего возникновения в прилегающих к ним участках базы областей $-l_{p}<x<x_{p}$ и $x_{n}<x<l_{n}$, почти свободных от неосновных носителей заряда. Эти области отделены от квазинейтральной электронно-дырочной плазмы с высокой плотностью, расположенной при $x_{p}<x<x_{n}$, крутыми диффузионными фронтами, движущимися навстречу друг другу, как изображено на рис. 2. Со временем их толщины и сопротивления увеличиваются, причем в дДРВ рано или поздно неизбежно ${ }^{2}$ нарушается условие квазинейтральности и вблизи границ $x_{n, p}$ возникают области пространственного заряда (ОПЗ) [26-28] (см. рис. 2), так как $N_{d}(x)-N_{a}(x) \rightarrow 0$ при $x \rightarrow 0$. СВОП завершается, когда границы $x_{n, p}$ встречаются при $t=T_{b}$, ДРВ практически полностью ${ }^{3}$ освобождается от неравновесных носителей заряда, ток индуктивного накопителя $I=I_{b}$, падение напряжения $U$ на ДРВ и нагрузке $R$ равно $U_{p p} \equiv U\left(T_{b}\right)$, а ток через ДРВ $I_{d} \equiv I_{b}-U / R=I_{d b}$.

\footnotetext{
${ }^{2}$ В этом состоит принципиальное отличие дДРВ от эДРВ, в которых ОПЗ возникают во время СВОП только при достаточно больших плотностях тока (см. разд. 2 в работе [25]).

${ }^{3}$ На самом деле относительно небольшое количество неравновесных носителей заряда (главным образом дырок) остается в дДРВ, в том числе - в ОПЗ, даже после начала обрыва тока. Например, в случае, изображенном на рис. 2, концентрация электронов и дырок в ОПЗ равна $10^{13} \mathrm{~cm}^{-3}$ при $t=T_{U M}$, вследствие чего через ОПЗ протекает ток проводимости $\sim 30 \mathrm{~A}$, шунтирующий барьерную емкость $p-n$-перехода.
} 

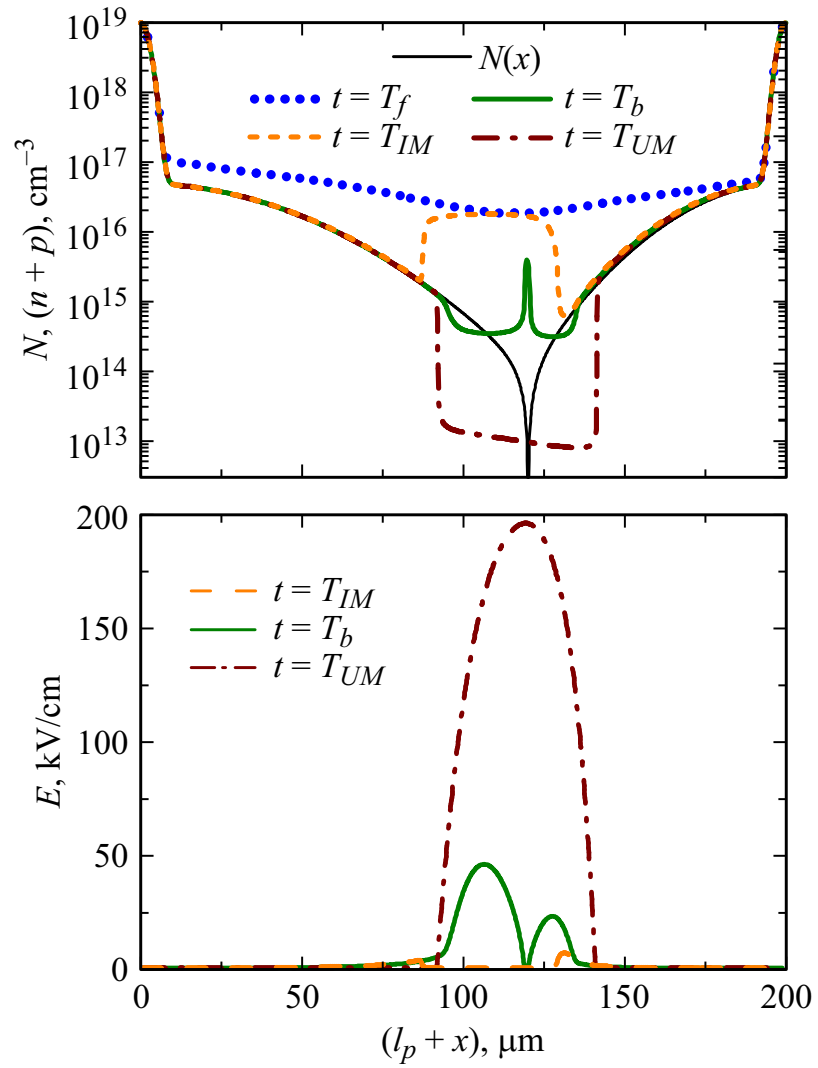

Рис. 2. Распределения легирующих примесей $N$ (тонкие линии), свободных носителей заряда $(n+p)$ и напряженности поля $E$ (толстые линии) по толщине дДРВ с $S=2 \mathrm{~cm}^{2}$ и $l_{p}=122$ мкм в моменты времени $t=T_{I M}, T_{b}, T_{U M}$.

После этого начинается СОТ, во время которой нелинейная барьерная емкость ДРВ заряжается до максимального напряжения $U_{M} \gg U_{p p}$ в момент $t=T_{U M}$ за время $t_{b}=T_{U M}-T_{b} \ll t_{r}$. Типичные ампер-секундные и вольтсекундные характеристики дДРВ во время СВОП и СОТ показаны сплошными линиями на рис. 3.

Важнейшим параметром, характеризующим эффективность работы любых ДРВ, является энергия $W_{d}$, рассеиваемая прибором за один цикл коммутации. В работах $[1,16,22]$ было высказано предположение, что для минимизации $W_{d}$ необходимо такое согласование параметров импульсов прямого и обратного токов с параметрами $p^{+}-p-n-n^{+}$-структур, при котором встреча границ $x_{n, p}$ происходит в плоскости $p-n$-перехода $x=0$, как в случае, изображенном на рис. 2. Иными словами, должно быть выполнено условие

$$
x_{p} \rightarrow-0 \quad \text { и } \quad x_{n} \rightarrow+0 \quad \text { при } \quad t \rightarrow T_{b} .
$$

Оно представляется вполне очевидным для эДРВ, так как исключает появление ОПЗ вблизи $p-n$-перехода во время СВОП и вызваемое этим резкое увеличение напряжения паразитного предымпульса $U_{p p} \equiv U\left(T_{b}\right)$ при достаточно малой плотности обрываемого тока $J_{d b}=I_{d b} / S$ (см. [16] и разд. 2 в работе [25]). В дДРВ, как было уже отмечено, ОПЗ возникает даже при малых $J_{d b}$, однако и в этом случае выполнение (2) с достаточной для практических целей точностью обеспечивает минимум $W_{d}$ и одновременно максимумы $I_{b}, U_{M}$ при прочих равных условиях. Справедливость этого утверждения
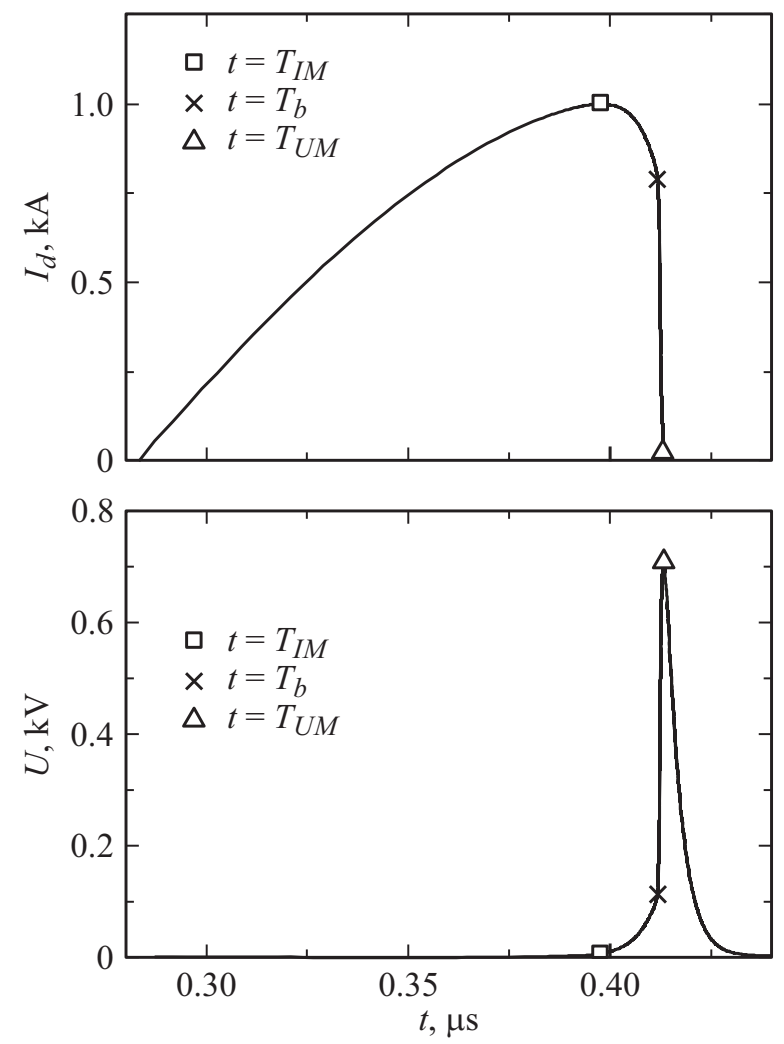

Рис. 3. Ампер-секундные и вольт-секундные характеристики дДРВ с $S=2.0 \mathrm{~cm}^{2}$ и $l_{p}=122$ мкм. Символами отмечены значения $I_{d}$ и $U$ в моменты времени $T_{I M}$ (квадраты), $T_{b}$ (кресты) и $T_{U M}$ (треугольники).

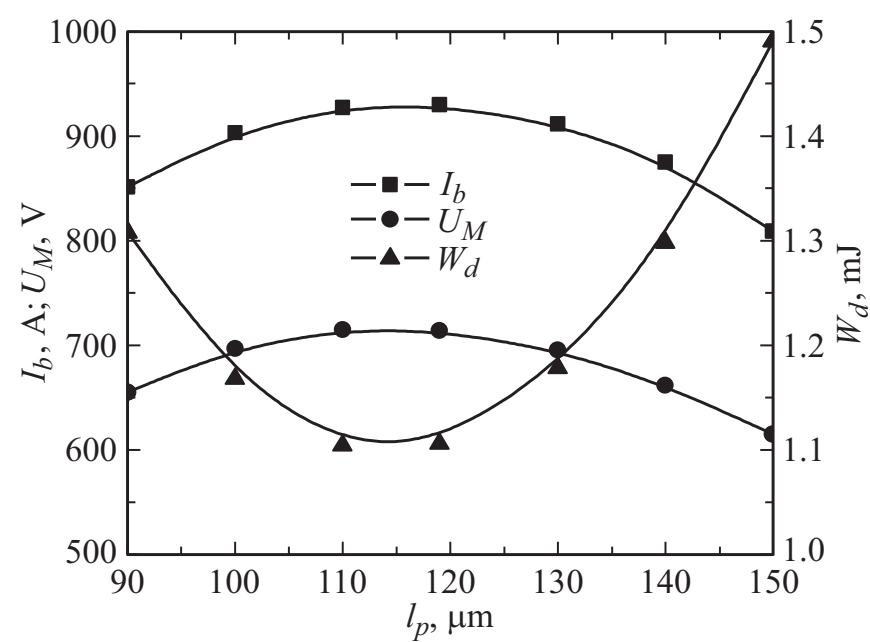

Рис. 4. Зависимости обрываемого тока $I_{b}$, амплитуды импульса напряжения $U_{M}$ и полной энергии потерь $W_{d}$ в дДРВ с площадью $S=2.5 \mathrm{~cm}^{2}$ от глубины залегания $p-n$-перехода $l_{p}$ при $U_{\mathrm{Cr}}=52$ В. Условие (2) выполняется при $l_{p}=120$ мкм. 
подтверждается результатами моделирования, пример которых приведен на рис. 4.

В следующих подразделах изложены результаты двух серий численных экспериментов, которые позволили прояснить влияние всех физических эффектов на работу дДРВ. В обеих сериях подбирались такие значения $l_{p}$, которые обеспечивали выполнение условия (2) с точностью 1-2 мкм.

\section{1. Зависимость параметров импульса от площади дДРВ}

В первой серии изучались зависимости параметров импульса от площади прибора $S$ при амплитуде $U_{M} \approx 710 \mathrm{~B}$, постоянство которой поддерживалось за счет подходящего выбора напряжений $U_{C f}=-U_{\mathrm{Cr}}$. Зависимости $U_{\mathrm{Cr}}(S)$ и $l_{p}(S)$ показаны на рис. 5 .

Результаты обработки ампер-секундных и вольтсекундных характеристик, полученных в этой серии численных экспериментов, приведены на рис. 6 вместе с результатами расчетов по формулам (II.6), (II.24), (II.25) и (II.26). Входящая в них величина обрываемого тока $I_{b}$ вычислялась по обращенной формуле (II.24)

$$
I_{b}=\frac{U_{B}}{R}\left[\frac{U_{M}}{b U_{B}}\left(\frac{L_{r}}{R^{2} C_{B}}\right)^{k \frac{1-\beta}{k-1}}\right]^{1 / \beta}
$$

при $U_{M}=710 \mathrm{~B}$, где $C_{B}=\varepsilon E_{B} S / U_{B}$ - дифференциальная емкость дДРВ, а $E_{B}-$ максимальная напряженность поля при $U=U_{B}, k=3 / 2, b$ и $\beta$ - подгоночные параметры [25]

Необходимо отметить, что столь замечательное количественное совпадение результатов численного моделирования и аналитических расчетов получилось в результате небольшой корректировки подгоночных параметров, входящих в формулы (II.24), (II.25) и (II.26), которая заключалась в заменах $b \rightarrow 0.9 b, a \rightarrow 1.18 a$ и $g \rightarrow 1.18 g$. Фактически это означает, что погрешность

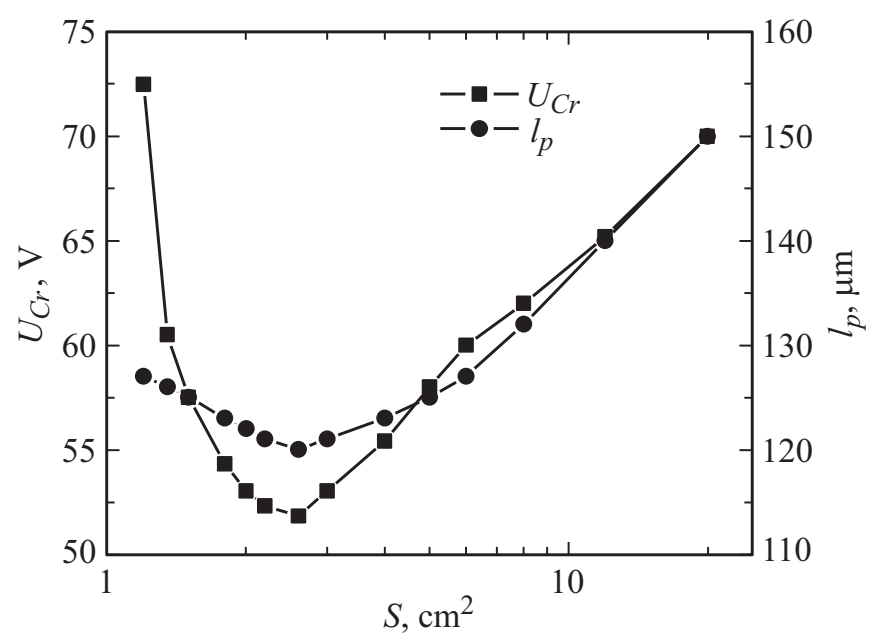

Рис. 5. Зависимости величин $l_{p}(S)$ и $U_{\mathrm{Cr}}(S)$, при которых выполняется условие $(2)$ и амплитуда импульса $U_{M} \approx 710 \mathrm{~B}$.

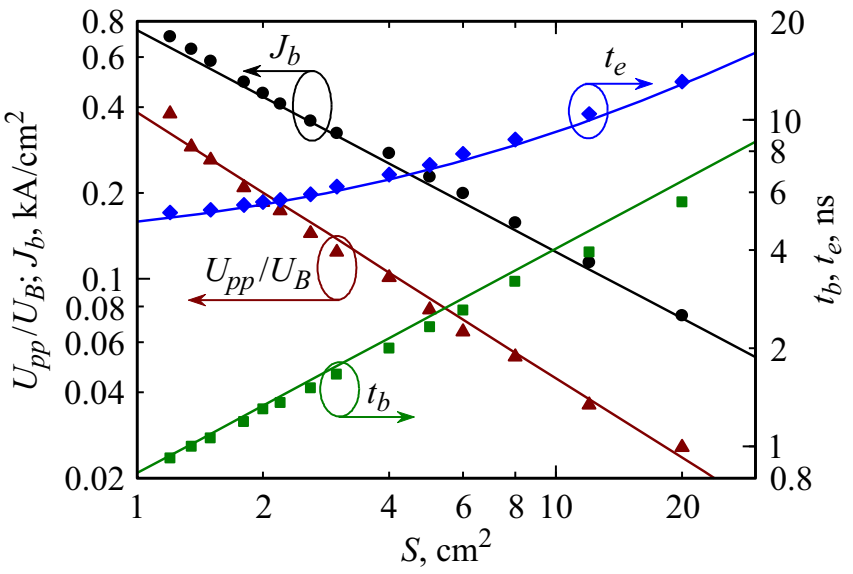

Рис. 6. Зависимости напряжения предымпульса $U_{p p}$, плотности обрываемого тока $J_{b}$ и длительности фронта $t_{b}$ и импульса $t_{e}$ от площади дДРВ при $U_{M}=710 \mathrm{~B}$, полученные путем обработки результатов численного моделирования (символы). Линии - расчет по формулам (II.6), (II.24), (II.25) и (II.26) при скорректированных значениях параметров $a, b$ и $g$ (см. текст).

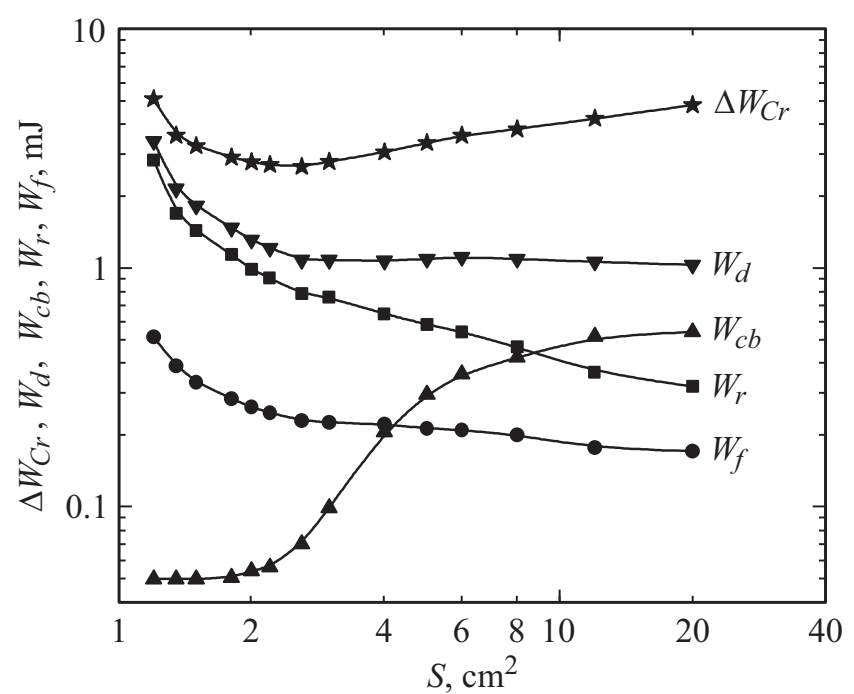

Рис. 7. Зависимости энергий $W_{d}, W_{f}, W_{r}$ и $W_{c b}$ от площади дДРВ $S$ при $U_{M}=710 \mathrm{~B}$.

аналитической теории [25] равна 10\% при расчете амплитуды и $18 \%$ при расчете длительностей импульса.

При малых $S$ (больших $J_{b}$ ) эта погрешность возникает из-за того, что теория [25] не учитывает наличие двух ОПЗ вблизи с $p$-n-переходом в момент $t=T_{b}$ (см. pис. 2), падение напряжения на которых $U_{p p}$ соизмеримо с $U_{M}$ (см. рис. 3,6$)$. При больших $S$ (малых $J_{b}$ ) основной причиной погрешности является наличие остаточных неравновесных носителей заряда даже при $t>T_{b}$ (см. сноску 3). Затягивание этих носителей заряда в ОПЗ приводит к возникновению заметного тока проводимости, шунтирующего барьерную емкость обратно смещенного дДРВ и таким образом замедляющего нарастание напряжения в время COT. 
Еще одним важнейшим параметром, характеризующим эффективность генераторов, является энергия $W_{d}$, рассеиваемая ДРВ за один цикл коммутации. Она складывается из энергий $W_{f}, W_{r}$ и $W_{c b}$, рассеиваемых во время СПН, СВОП и СОТ соответственно. Зависимости всех этих энергий от $S$, изображенные на рис. 7, позволяют сделать следующие выводы. Во-первых, энергии $W_{f}, W_{r}$, как и следовало ожидать, монотонно уменьшаются с ростом $S$ и падением $J_{b}$. Во-вторых, увеличение концентрации остаточных неравновесных носителей заряда за пределами области $x_{p}<x<x_{n}$ с ростом $S$ приводит к сильному возрастанию энергии $W_{c b}$, pacceиваемой во время СОТ, так что полная энергия $W_{d}$, рассеиваемая ДРВ, практически не зависит от $S$ при $S>3 \mathrm{~cm}^{2}$. В-третьих, потери во время СПН дают заметный (15-20\%) вклад в $W_{d}$. В-четвертых, коэффициент полезного действия (кпд) генератора, приближенно равный $\left(1-W_{d} / \Delta W_{\mathrm{Cr}}\right)$ без учета потерь в цепи заряда емкостных накопителей $C_{f}, C_{r}$ и в первичных ключах $S W_{1,2}$, увеличивается с ростом $S$ от 0.34 до 0.79 (здесь $\Delta W_{\mathrm{Cr}}$ - уменьшение энергии в емкостном накопителе $C_{r}$ за один цикл коммутации).

\section{2. Зависимость параметров импульса от плотности обрываемого тока}

Во второй серии изучались зависимости параметров импульса от плотности обрываемого тока $J_{b}=J_{b} / S$ при фиксированных значениях $S=2.5 \mathrm{~cm}^{2}$ и $l_{p}=120$ мкм. Величина $I_{b}$ определялась напряжением $U_{\mathrm{Cr}}=-U_{C f}$, увеличение которого от $20 \mathrm{~B}$ до $100 \mathrm{~B}$ приводило к росту обрываемого тока по линейному закону $I_{b} \approx U_{\mathrm{Cr}} / r_{b}$. Сопротивление $r_{b}=5.78$ мОм превышало характеристическое сопротивление контура обратной накачки $\sqrt{L_{r} / C_{r}}=4.46$ мОм из-за потерь в дДРВ.

Результаты обработки ампер-секундных и вольтсекундных характеристик, полученных в этой серии численных экспериментов, приведены на рис. 8 вместе с результатами расчетов по формулам (II.6), (II.24), (II.25) и (II.26). Как видно, и в этом случае корректировка значений подгоночных параметров $a, b, g$ приводит к совпадению численных и аналитических результатов, но только при $J_{b}<400 \mathrm{~A} / \mathrm{cm}^{2}$, когда $U_{M}<800$ В. Зависимости энергий $W_{f}, W_{r}, W_{c b}$ и $W_{d}$ от $J_{b}$, изображенные на рис. 9, можно приближенно описать степенными функциями $W_{f}, W_{c b} \propto J_{b}^{3 / 2}$ и $W_{r}, W_{d} \propto J_{b}^{2}$. С ростом $J_{b}$ кпд генератора сначала медленно увеличивается, достигает максимума 0.6 при $J_{b} \approx 430 \mathrm{~A} / \mathrm{cm}^{2}$, после чего медленно уменьшается. Одновременно начинается резкий рост энергии $W_{c b}$ и качественно изменяются зависимости от $J_{b}$ остальных параметров импульса, как это изображено на рис. 8.

Причиной этого является резкое увеличение скорости лавинного размножения остаточных неравновесных электронов и дырок в ОПЗ при $U \rightarrow U_{B}$ и скачкообразный рост тока проводимости, шунтирующего барьерную емкость диода. При $U-U_{B} \ll U_{B}$ такой динамический

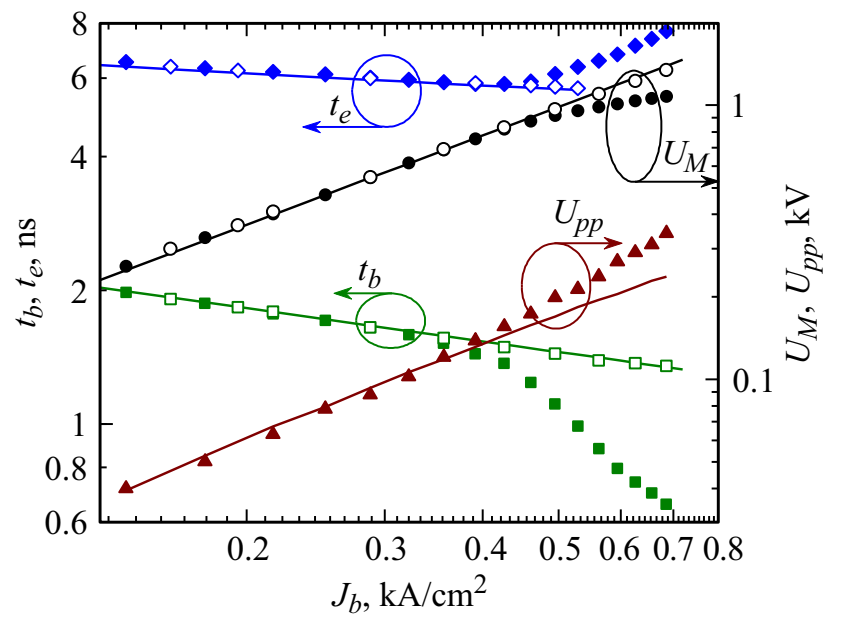

Рис. 8. Зависимости напряжения предымпульса $U_{p p}$, амплитуды $U_{M}$ и длительности фронта $t_{b}$ и импульса $t_{e}$ от плотности тока, обрываемого дДРВ с площадью $S=2.5 \mathrm{~cm}^{2}$, полученные путем обработки результатов моделирования с учетом (темные символы) и без учета (светлые символы) ударной ионизации Линии - расчет по формулам (II.6), (II.24), (II.25) и (II.26) при скорректированных значениях параметров $a, b$ и $g$.

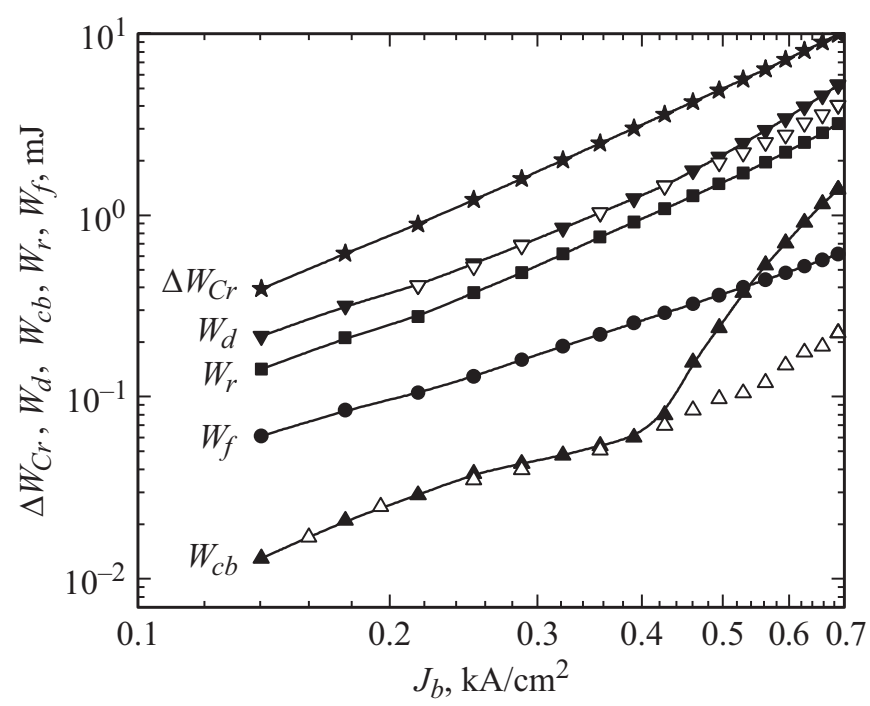

Рис. 9. Зависимости $W_{d}, W_{f}, W_{r}$ и $W_{c b}$ от плотности обрываемого тока $J_{b}$, полученные путем обработки результатов моделирования с учетом (темные символы) и без учета (светлые символы) ударной ионизации.

лавинный пробой просто „срезает“ вершину колоколообразного импульса напряжения, уменьшая его амплитуду и длительность фронта. Однако дальнейший рост перенапряжения приводит еще к возникновению осцилляций на вершине импульса, изображенных на рис. 10. Похожие осцилляции наблюдались ранее в работах [29-31] и объяснялись авторами IMPATT эффектом (IMPact Avalanch Transit Time). Следует подчеркнуть, что ударная ионизация практически не влияет на зависимость $U(t)$ вплоть до $U=U_{M}>U_{B}$. Поэтому сильное сокращение 


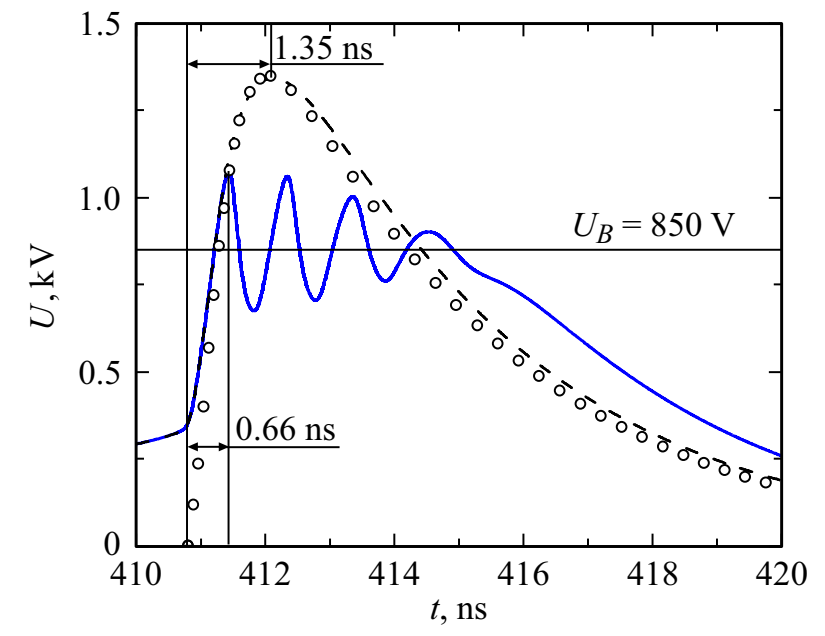

Рис. 10. Вольт-секундные характеристики дДРВ с площадью $S=2.5 \mathrm{~cm}^{2}$ при $U_{\mathrm{Cr}}=100 \mathrm{~B}$ и $J_{b}=700 \mathrm{~A} / \mathrm{cm}^{2}$, полученные путем численного моделирования с учетом (сплошная линия) и без учета (штриховая линия) ударной ионизации. Символы решение задачи Коши (II.20), (II.21), уменьшенное на 10\%.

длительности фронта $t_{b}$ объясняется просто тем, что меньшая амплитуда импульса со „срезанной“ за счет пробоя вершиной достигается за меньшее время (см. рис. 10).

\section{4. Заключение}

В заключение обсудим, каким образом можно охарактеризовать эффективность работы ДРВ в качестве прерывателей тока в генераторах импульсов с промежуточным индуктивным накопителем энергии. Прежде всего отметим, что генератор предназначен для формирования на нагрузке импульсов с заданными величинами перепа-

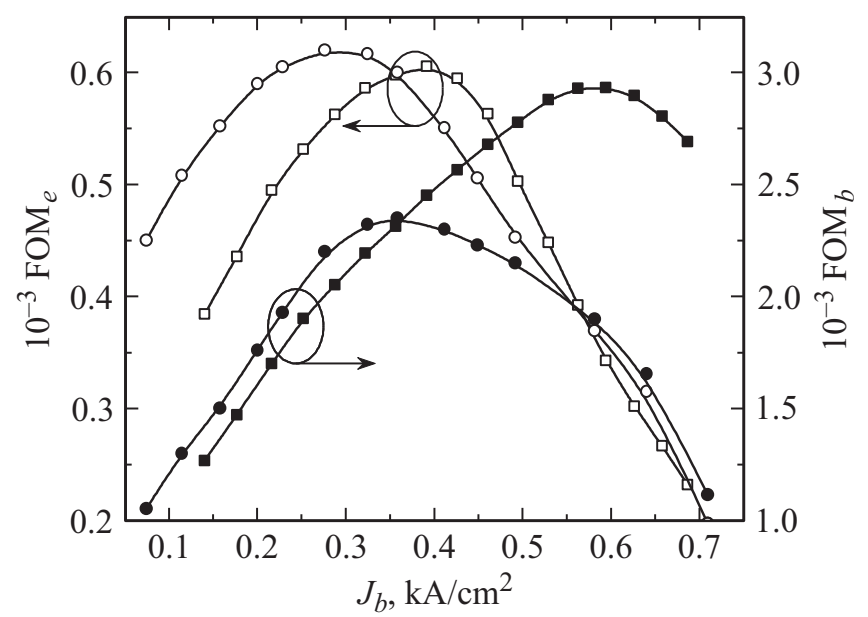

Рис. 11. Зависимости показателей качества дДРВ $\mathrm{FOM}_{e}$ (светлые символы) и $\mathrm{FOM}_{b}$ (темные символы) от плотности обрываемого тока $J_{b}$, полученные в первой (кружки) и второй (квадраты) сериях численных экспериментов. да напряжения $\left(U_{M}-U_{p p}\right)$, длительности фронта $t_{b}$ или, в зависимости от назначения генератора, длительности импульса $t_{e}$ и частотой повторения $f$. Предельная частота $f_{\text {max }}$ определяется не только потерями в самом ДРВ (т. е. энергией $W_{d}$ ), но и энергией коммутационных потерь $W_{s w}$ в первичных ключах $S W_{1.2}$ [32]. Конечно, энергия $W_{s w}$ зависит от типа ключей $S W_{1.2}$, но в любом случае она существенно увеличивается с ростом коммутируемого напряжения $\left(U_{\mathrm{Cr}}\right)$ и с уменьшением времени коммутации, которое должно быть много меньше $t_{r}$. Поэтому представляется целесообразным использовать обобщенный показатель качества (Figure Of Merit) ДРВ в виде

$$
\mathrm{FOM}_{i}=K_{U} K_{t i} K_{W},
$$

где $K_{U}=\left(U_{M}-U_{p p}\right) / U_{\mathrm{Cr}}-$ коэффициент усиления напряжения, $K_{t i}=t_{r} / t_{i}$ - коэффициент обострения $(i=b)$ или сжатия $(i=e)$ импульса, $K_{W}=\Delta W_{\mathrm{Cr}} / W_{d}$ коэффициент потерь энергии в ДРВ. Примеры зависимостей $\mathrm{FOM}_{e, b}$ от плотности обрываемого тока $J_{b}$, приведенные на рис. 11, указывают на возможность использования такого показателя качества для оптимизации параметров ДРВ и режима его работы, а также для сравнения эффективности ДРВ различных типов.

\section{Благодарности}

Автор благодарен С.Н. Юркову и Т.Т. Мнацаканову, предоставившим возможность провести моделирование с помощью программы „Исследование“.

\section{Финансирование работы}

Работа выполнена при поддержке РФФИ (грант № 16-08-01292).

\section{Список литературы}

[1] И.В. Грехов, Г.А. Месяц. УФН, 175 (7), 735 (2005).

[2] И.В. Грехов, В.М. Тучкевич. Новые принципы коммутации больших мощностей полупроводниковыми приборами (Л., Наука, 1988).

[3] A.F. Kardo-Sysoev. In: Ultra-Wideband Radar Technology, ed. by J.D. Taylor (Boca Raton-London-N.Y.-Washington, CRS Press, 2001) Chap. 9.

[4] И.В. Грехов, В.М. Ефанов, А.Ф. Кардо-Сысоев, С.В. Шендерей. Письма ЖТФ, 9 (7), 435 (1983).

[5] С.И. Зиенко. Приборы и техника эксперимента, 27 (4), 100 (1984).

[6] I.V. Grekhov, V.M. Efanov, A.F. Kardo-Sysoev, S.V. Shenderey. Solid-State Electron., 28 (6), 597 (1985).

[7] А.Ф. Кардо-Сысоев, И.Г. Чашников. Приборы и техника эксперимента, 29 (1), 95 (1986).

[8] В.И. Брылевский, И.В. Грехов, В.М. Ефанов, А.Ф. КардоСысоев, И.Г. Чашников, Д.И. Шеметило. ЖТФ, 58 (11), 2244 (1988).

[9] В.С. Белкин, Г.И. Шульженко. Формирователи мощных наносекундных и пикосекундных импульсов на полупроводниковой элементной базе (ИЯФ СО АН СССР, 1991), Препринт 91-51. 
[10] V.S. Belkin, G.I. Shulzchenko. Rev. Sci. Instrum., 65, 751 (1994).

[11] V.I. Brylevsky, V.M. Efanov, A.F. Kardo-Sysyev, I.G. Tchashnicov. Proc. XXIIInt. Power Modulator Symp. (Boca Raton, FL, USA, 1996) p. 51.

[12] И.В. Грехов, А.Г. Люблинский, И.А. Смирнова. ЖТФ, 85 (11), 104 (2015).

[13] В.М. Ефанов, А.Ф. Кардо-Сысоев, И.А. Смирнова. ФТП, 21 (4), 620 (1987).

[14] И.В. Грехов, А.С. Кюрегян, Т.Т. Мнацаканов, С.Н. Юрков. ФТП, 37 (9), 1148 (2003).

[15] I.V. Grekhov, P.A. Ivanov, D.V. Khristyuk, A.O. Konstantinov, S.V. Korotkov, T.P. Samsonova. Solid-State Electron., 47, 1769 (2003).

[16] А.С. Кюрегян. ЖТФ, 74 (6), 57 (2004).

[17] X. Yang, Y. Li, H. Wang, Z. Li, Z. Ding. J. Appl. Phys., 109, 014917 (2011)

[18] Y. Sharabani, Y. Rosenwaks, D. Eger. Phys. Rev. Appl., 4, 014015 (2015)

[19] П.А. Иванов, И.В. Грехов. ЖТФ, 85 (6), 111 (2015).

[20] A.S. Kesar, Y. Sharabani, I. Shafir, S. Zoran, A. Sher. IEEE Trans. Plasma Sci., 44, 2424 (2016).

[21] M.J. Chudobiak, D.J. Walkey. Electron. Lett., 32 (16), 1513 (1996).

[22] А.С. Кюрегян. Патент РФ № 2197034, кл. Н 01 L 29/861, Приоритет от 30.07.2001, публ. 20.01.2003 Бюл. № 2.

[23] П.В. Васильев, С.К. Любутин, А.В. Пономарев, С.Н. Рукин, Б.Г. Словиковский, С.Н. Цыранов, С.О. Чолах. ФТП, 43 (7), 985 (2009).

[24] T.T. Mnatsakanov, I.L. Rostovtsev, N.I. Philatov. Solid-State Electron., 30 (6), 579 (1987).

[25] А.С. Кюрегян. ФТП, 53 (7), 986 (2019).

[26] H. Benda, E. Spenke. Proc. IEEE, 55 (8), 1331 (1967).

[27] H. Benda, F. Dannhauser. Solid State Electron., 11, 1 (1968).

[28] И.В. Грехов, А.С. Кюрегян. ЖТФ, 75 (7), 88 (2005).

[29] В.А. Козлов, А.Ф. Кардо-Сысоев, В.И. Брылевский. ФТП, 35 (5), 629 (2001).

[30] В.А. Козлов, А.В. Рожков, А.Ф. Кардо-Сысоев. ФТП, 37 (12), 1480 (2003).

[31] С.А. Дарзнек, С.К. Любутин, С.Н. Рукин, Б.Г. Словиковский. ФТП, 36 (5), 629 (2002).

[32] L.M. Merensky, A.F. Kardo-Sysoev, D. Shmilovitz, A.S. Kesar. IEEE Trans. Plasma Sci., 41 (11), 3138 (2013).

Редактор А.Н. Смирнов

\section{High voltage diffused step recovery diodes. I. Numerical simulation}

\section{A.S.Kyuregyan \\ NPO Energomodule, 109052 Moscow, Russia}

Abstract The operation of diffusion step recovery diodes (dSRD) as current interrupters in high-power nanosecond pulse generators was studied in detail by the methods of numerical computer simulation for the first time. One of the necessary conditions that minimize th loss in dSRD was specified. Dependence of pre-pulse voltage, front duration, amplitude and duration of pulse formed on the resistive load, and the energy of switching losses in the dSRD on the device area and the break current density are obtained. It is shown that simulation results can be described by simple analytical formulas obtained in the second part of the work, with an accuracy of $10-20 \%$ if the pulse amplitude does not exceed the avalanche breakdown voltage of the dSRD. The generalized figure of merit which can be used for optimization of dSRD parameters, a mode of its operation and comparison of effeciency of current interrupters of various types was offered. 